\title{
Strong Convergence Theorems for Family of Nonexpansive Mappings and System of Generalized Mixed Equilibrium Problems and Variational Inequality Problems
}

\author{
Yekini Shehu \\ Mathematics Institute, African University of Science and Technology, Abuja, Nigeria \\ Correspondence should be addressed to Yekini Shehu, deltanougt2006@yahoo.com \\ Received 20 October 2010; Revised 5 January 2011; Accepted 8 February 2011 \\ Academic Editor: A. Zayed \\ Copyright (C) 2011 Yekini Shehu. This is an open access article distributed under the Creative \\ Commons Attribution License, which permits unrestricted use, distribution, and reproduction in \\ any medium, provided the original work is properly cited. \\ We introduce a new iterative scheme by hybrid method for finding a common element of the set \\ of common fixed points of infinite family of nonexpansive mappings, the set of common solutions \\ to a system of generalized mixed equilibrium problems, and the set of solutions to a variational \\ inequality problem in a real Hilbert space. We then prove strong convergence of the scheme to a \\ common element of the three sets. We give some applications of our results. Our results extend \\ important recent results.
}

\section{Introduction}

Let $K$ be a nonempty closed and convex subset of a real Hilbert space $H$. A mapping $A$ : $K \rightarrow H$ is called monotone if

$$
\langle A x-A y, x-y\rangle \geq 0, \quad \forall x, y \in K
$$

The variational inequality problem is to find an $x^{*} \in K$ such that

$$
\left\langle y-x^{*}, A x^{*}\right\rangle \geq 0, \quad \forall y \in K
$$

(see, e.g., [1]). We will denote the set of solutions to the variational inequality problem (1.2) by $\operatorname{VI}(K, A)$. 
A mapping $A: K \rightarrow H$ is called inverse-strongly monotone (see, e.g., $[2,3]$ ) if there exists a positive real number $\alpha$ such that $\langle A x-A y, x-y\rangle \geq \alpha\|A x-A y\|^{2}$, for all $x, y \in K$. For such a case, $A$ is called $\alpha$-inverse-strongly monotone.

A mapping $T: K \rightarrow K$ is said to be nonexpansive if

$$
\|T x-T y\| \leq\|x-y\|
$$

for all $x, y \in K$. A point $x \in K$ is called a fixed point of $T$ if $T x=x$. The set of fixed points of $T$ is the set $F(T):=\{x \in K: T x=x\}$.

Let $\varphi: K \rightarrow \mathbb{R}$ be a real-valued function and $A: K \rightarrow H$ a nonlinear mapping. Suppose that $F: K \times K$ into $\mathbb{R}$ is an equilibrium bifunction. That is, $F(u, u)=0$, for all $u \in K$. The generalized mixed equilibrium problem is to find $x \in K$ (see, e.g., [4-6]) such that

$$
F(x, y)+\varphi(y)-\varphi(x)+\langle A x, y-x\rangle \geq 0
$$

for all $y \in K$. We will denote the set of solutions of this generalized mixed equilibrium problem by $\operatorname{GMEP}(F, A, \varphi)$. Thus

$$
\operatorname{GMEP}(F, A, \varphi):=\left\{x^{*} \in K: F\left(x^{*}, y\right)+\varphi(y)-\varphi\left(x^{*}\right)+\left\langle A x^{*}, y-x^{*}\right\rangle \geq 0, \forall y \in K\right\} .
$$

If $\varphi=0, A=0$, then problem (1.4) reduces to equilibrium problem studied by many authors (see, e.g., [7-14]), which is to find $x^{*} \in K$ such that

$$
F\left(x^{*}, y\right) \geq 0,
$$

for all $y \in K$. The set of solutions of (1.6) is denoted by $\operatorname{EP}(F)$.

If $\varphi=0$, then problem (1.4) reduces to generalized equilibrium problem studied by many authors (see, e.g., [15-18]), which is to find $x^{*} \in K$ such that

$$
F\left(x^{*}, y\right)+\left\langle A x^{*}, y-x^{*}\right\rangle \geq 0
$$

for all $y \in K$. The set of solutions of (1.7) is denoted by $\operatorname{GEP}(F, A)$.

If $A=0$, then problem (1.4) reduces to mixed equilibrium problem considered by many authors (see, e.g., [19-21]), which is to find $x^{*} \in K$ such that

$$
F\left(x^{*}, y\right)+\varphi(y)-\varphi\left(x^{*}\right) \geq 0
$$

for all $y \in K$. The set of solutions of (1.8) is denoted by $\operatorname{MEP}(F, \varphi)$.

The generalized mixed equilibrium problems include fixed point problems, optimization problems, variational inequality problems, Nash equilibrium problems, and equilibrium problems as special cases (see, e.g., [22]). Numerous problems in physics, optimization, and economics reduce to find a solution of problem (1.4). Several methods have been proposed to solve the fixed point problems, variational inequality problems, and equilibrium problems in the literature. See, for example, [23-33]. 
One of the iterative processes (see Halpern [34]) which is often used to approximate a fixed point of a nonexpansive mapping $T$ is defined as follows. Take an initial guess $x_{0} \in K$ arbitrarily and define $\left\{x_{n}\right\}$ recursively by

$$
x_{n+1}=\alpha_{n} x_{0}+\left(1-\alpha_{n}\right) T x_{n}, \quad n \geq 0,
$$

where $\left\{\alpha_{n}\right\}$ is a sequence in $[0,1]$. The iteration process (1.9) has been proved to be strongly convergent both in Hilbert spaces [34-36] and uniformly smooth Banach spaces [37,38] when the sequence $\left\{\alpha_{n}\right\}$ satisfies the conditions

(i) $\lim _{n \rightarrow \infty} \alpha_{n}=0$,

(ii) $\sum_{n=1}^{\infty} \alpha_{n}=\infty$,

(iii) either $\sum_{n=1}^{\infty}\left|\alpha_{n+1}-\alpha_{n}\right|<\infty$ or $\lim _{n \rightarrow \infty} \alpha_{n} / \alpha_{n+1}=1$.

Motivated by (1.9), Martinez-Yanes and Xu [39] introduced the following iterative scheme for a single nonexpansive mapping $T$ in a Hilbert space:

$$
\begin{gathered}
x_{0} \in K, \\
y_{n}=\alpha_{n} x_{0}+\left(1-\alpha_{n}\right) T x_{n}, \\
C_{n}=\left\{z \in K:\left\|y_{n}-z\right\|^{2} \leq\left\|x_{n}-z\right\|^{2}+\alpha_{n}\left(\left\|x_{0}\right\|^{2}+2\left\langle x_{n}-x_{0}, z\right\rangle\right)\right\}, \\
Q_{n}=\left\{z \in K:\left\langle x_{n}-z, x_{n}-x_{0}\right\rangle \leq 0\right\}, \\
x_{n+1}=P_{C_{n} \cap Q_{n}} x_{0},
\end{gathered}
$$

where $P_{K}$ denotes the metric projection of $H$ onto a closed and convex subset $K$ of $H$. They proved that if $\left\{\alpha_{n}\right\} \subset(0,1)$ and $\lim _{n \rightarrow \infty} \alpha_{n}=0$, then the sequence $\left\{x_{n}\right\}$ converges strongly to $P_{F(T)} x_{0}$.

Furthermore, algorithm (1.10) has been modified by many authors for relatively nonexpansive mappings and quasi- $\phi$-nonexpansive mappings in Banach spaces (see, e.g., [4043]).

Recently, Ceng and Yao [44] introduced a new iterative scheme of approximating a common element of the set of solutions to mixed equilibrium problem and set of common fixed points of finite family of nonexpansive mappings in a real Hilbert space $H$. In the proof process of their results, they imposed the following condition on a nonempty closed and convex subset $K$ of $H$ :

(E) $A: K \rightarrow \mathbb{R}$ is $\eta$-strongly convex and its derivative $A^{\prime}$ is sequentially continuous from weak topology to the strong topology.

We remark here that this condition (E) has been used by many authors for approximation of solution to mixed equilibrium problem in a real Hilbert space (see, e.g., $[45,46]$ ). However, it is observed that condition (E) does not include the case $A(x)=\|x\|^{2} / 2$ and $\eta(x, y)=x-y$. Furthermore, Peng and Yao [19],Wangkeeree and Wangkeeree [47], and other 
authors replaced condition (E) with these conditions:

(B1) for each $x \in H$ and $r>0$, there exist a bounded subset $D_{x} \subseteq K$ and $y_{x} \in K$ such that, for any $z \in K \backslash D_{x}$

$$
F\left(z, y_{x}\right)+\varphi\left(y_{x}\right)-\varphi(z)+\frac{1}{r}\left\langle y_{x}-z, z-x\right\rangle<0,
$$

or

(B2) $K$ is a bounded set.

Consequently, conditions (B1) and (B2) have been used by many authors in approximating solution to generalized mixed equilibrium (mixed equilibrium) problems in a real Hilbert space (see, e.g., $[19,47])$. method.

In [48], Takahashi et al. proved the following convergence theorem using hybrid

Theorem 1.1 (Takahashi et al. [48]). Let $K$ be a nonempty closed and convex subset of a real Hilbert space $H$. Let $T$ be a nonexpansive mapping of $K$ into itself such that $F(T) \neq \emptyset$. For $C_{1}=K, x_{1}=$ $P_{C_{1}} x_{0}$, define sequences $\left\{x_{n}\right\}_{n=0}^{\infty}$ and $\left\{y_{n}\right\}_{n=1}^{\infty}$ of $K$ as follows:

$$
\begin{gathered}
y_{n}=\alpha_{n} x_{n}+\left(1-\alpha_{n}\right) T x_{n}, \quad n \geq 1, \\
C_{n+1}=\left\{z \in C_{n}:\left\|y_{n}-z\right\| \leq\left\|x_{n}-z\right\|\right\}, \quad n \geq 1, \\
x_{n+1}=P_{C_{n+1}} x_{0}, \quad n \geq 1 .
\end{gathered}
$$

Assume that $\left\{\alpha_{n}\right\}_{n=1}^{\infty} \subset[0,1)$ satisfies $0 \leq \alpha_{n}<\alpha<1$. Then, $\left\{x_{n}\right\}_{n=0}^{\infty}$ converges strongly to $P_{F(T)} x_{0}$.

Motivated by the results of Takahashi et al. [48], Kumam [49] studied the problem of approximating a common element of set of solutions to an equilibrium problem, set of solutions to variational inequality problem, and set of fixed points of a nonexpansive mapping in a real Hilbert space. In particular, he proved the following theorem.

Theorem 1.2 (Kumam [49]). Let $K$ be a nonempty closed convex subset of a real Hilbert space $H$. Let $F$ be a bifunction from $K \times K$ satisfying $(A 1)-(A 4)$, and let $B$ be a $\beta$-inverse-strongly monotone mapping of $K$ into $H$. Let $T$ be a nonexpansive mapping of $K$ into $H$ such that $F(T) \cap \operatorname{EP}(F) \cap$ $\operatorname{VI}(K, B) \neq \emptyset$. For $C_{1}=K, x_{1}=P_{C_{1}} x_{0}$, define sequences $\left\{x_{n}\right\}_{n=0}^{\infty}$ and $\left\{z_{n}\right\}_{n=1}^{\infty}$ of $K$ as follows:

$$
\begin{array}{cc}
F\left(z_{n}, y\right)+\frac{1}{r_{n}}\left\langle y-z_{n}, z_{n}-x_{n}\right\rangle \geq 0, \quad \forall y \in K, \\
y_{n}=\alpha_{n} x_{n}+\left(1-\alpha_{n}\right) T P_{K}\left(z_{n}-\lambda_{n} B z_{n}\right), \quad n \geq 1, \\
C_{n+1}=\left\{z \in C_{n}:\left\|y_{n}-z\right\| \leq\left\|x_{n}-z\right\|\right\}, \quad n \geq 1, \\
x_{n+1}=P_{C_{n+1}} x_{0}, \quad n \geq 1 .
\end{array}
$$

Assume that $\left\{\alpha_{n}\right\}_{n=1}^{\infty} \subset[0,1),\left\{r_{n}\right\}_{n=1}^{\infty} \subset(0, \infty)$, and $\left\{\lambda_{n}\right\}_{n=1}^{\infty} \subset[0,2 \beta]$ satisfy

$$
\liminf _{n \rightarrow \infty} r_{n}>0, \quad 0<c \leq \lambda_{n} \leq f<2 \beta, \quad \lim _{n \rightarrow \infty} \alpha_{n}=0 .
$$

Then, $\left\{x_{n}\right\}_{n=0}^{\infty}$ converges strongly to $P_{F(T) \cap \mathrm{EP}(F) \cap \mathrm{VI}(K, B)} x_{0}$. 
Quite recently, Chantarangsi et al. [50] proved the following convergence theorem for approximation of fixed point of a nonexpansive mapping which is also a common solution to a system of generalized mixed equilibrium problems and variational inequality problem in a real Hilbert space.

Theorem 1.3 (Chantarangsi et al. [50]). Let $K$ be a nonempty closed and convex subset of a real Hilbert space $H$. Let $\theta_{1}, \theta_{2}$ be bifunctions from $K \times K$ satisfying $(A 1)-(A 4), \Psi_{1}$ an $\xi$-inversestrongly monotone mapping of $K$ into $H, \Psi_{2}$ a $\beta$-inverse-strongly monotone mapping of $K$ into $H$ with assumption (B1) or (B2), and $T: K \rightarrow K$ a nonexpansive mapping. Let $B$ be an $\omega$-Lipschitz continuous and relaxed $(v, v)$ co-coercive mapping of $K$ into $H, f: K \rightarrow K$ a contraction mapping with coefficient $\eta \in(0,1)$, and A a strongly positive linear bounded selfadjoint operator with coefficient $\bar{\gamma}>0$ and $0<\gamma<\bar{\gamma} / \eta$. Suppose that $F:=F(T) \cap \operatorname{GMEP}\left(\theta_{1}, \varphi, \Psi_{1}\right) \cap \operatorname{GMEP}\left(\theta_{2}, \varphi, \Psi_{2}\right) \cap$ $\mathrm{VI}(K, B) \neq \emptyset$. Let $\left\{z_{n}\right\}_{n=1}^{\infty},\left\{u_{n}\right\}_{n=1}^{\infty},\left\{y_{n}\right\}_{n=1}^{\infty}$, and $\left\{x_{n}\right\}_{n=1}^{\infty}$ be generated by

$$
\begin{gathered}
u_{n}=T_{r_{n}}^{\left(\theta_{1}, \varphi\right)}\left(x_{n}-r_{n} \Psi_{1} x_{n}\right), \\
v_{n}=T_{\left.s_{n}, \varphi\right)}^{\left(\theta_{2}\right)}\left(u_{n}-s_{n} \Psi_{1} u_{n}\right), \\
z_{n}=P_{K}\left(v_{n}-\alpha_{n} B S v_{n}\right), \\
y_{n}=\varepsilon_{n} \gamma f\left(x_{n}\right)+\beta_{n} x_{n}+\left(\left(1-\beta_{n}\right) I-\varepsilon_{n} A\right) z_{n}, \\
x_{n+1}=\gamma_{n} x_{n}+\left(1-\gamma_{n}\right) y_{n}, \quad \forall n \geq 1,
\end{gathered}
$$

where $\left\{r_{n}\right\} \subset[a, b] \subset[0,2 \xi],\left\{s_{n}\right\} \subset[c, d] \subset[0,2 \beta],\left\{\gamma_{n}\right\} \subset[h, j] \subset(0,1),\left\{\gamma_{n}\right\},\left\{\varepsilon_{n}\right\},\left\{\beta_{n}\right\}$ are three sequences in $(0,1)$ satisfying the following conditions:

(C1) $\lim _{n \rightarrow \infty} \varepsilon_{n}=0$ and $\sum_{n=1}^{\infty} \varepsilon_{n}=\infty$,

(C2) $0<\liminf _{n \rightarrow \infty} \beta_{n} \leq \lim \sup _{n \rightarrow \infty} \beta_{n}<1$,

(C3) $0<\liminf _{n \rightarrow \infty} r_{n} \leq \lim \sup _{n \rightarrow \infty} r_{n}<2 \xi$ and $\lim _{n \rightarrow \infty}\left|r_{n+1}-r_{n}\right|=0$,

(C4) $0<\liminf _{n \rightarrow \infty} s_{n} \leq \lim \sup _{n \rightarrow \infty} s_{n}<2 \beta$ and $\lim _{n \rightarrow \infty}\left|s_{n+1}-s_{n}\right|=0$,

(C5) $\left\{\alpha_{n}\right\} \subset[e, g] \subset\left(0,\left(2\left(v-v \omega^{2}\right)\right) / \omega^{2}\right), v>v \omega^{2}$ and $\lim _{n \rightarrow \infty}\left|\alpha_{n+1}-\alpha_{n}\right|=0$.

Then, $\left\{x_{n}\right\}$ converges strongly to $z=P_{F}(\gamma f+(I-A))(z)$.

Motivated by the ongoing research and the above-mentioned results, we modify algorithm (1.10) and introduce $a$ new iterative scheme for finding a common element of the set of fixed points of an infinite family of nonexpansive mappings, the set of common solutions to a system of generalized mixed equilibrium problems, and the set of solutions to a variational inequality problem in a real Hilbert space. Furthermore, we show that our new iterative scheme converges strongly to a common element of the three sets. In the proof process of our results, we use conditions (B1) and (B2) mentioned above. Our result extends many important recent results. Finally, we give some applications of our results.

\section{Preliminaries}

Let $H$ be a real Hilbert space with inner product $\langle\cdot, \cdot\rangle$ and norm $\|\cdot\|$, and let $K$ be a nonempty closed and convex subset of $H$. The strong convergence of $\left\{x_{n}\right\}_{n=1}^{\infty}$ to $x$ is written $x_{n} \rightarrow x$ as $n \rightarrow \infty$. 
For any point $u \in H$, there exists a unique point $P_{K} u \in K$ such that

$$
\left\|u-P_{K} u\right\| \leq\|u-y\|, \quad \forall y \in K .
$$

$P_{K}$ is called the metric projection of $H$ onto $K$. We know that $P_{K}$ is a nonexpansive mapping of $H$ onto $K$. It is also known that $P_{K}$ satisfies

$$
\left\langle x-y, P_{K} x-P_{K} y\right\rangle \geq\left\|P_{K} x-P_{K} y\right\|^{2},
$$

for all $x, y \in H$. Furthermore, $P_{K} x$ is characterized by the properties $P_{K} x \in K$ and

$$
\left\langle x-P_{K} x, P_{K} x-y\right\rangle \geq 0,
$$

for all $y \in K$ and

$$
\left\|x-P_{K} x\right\|^{2} \leq\|x-y\|^{2}-\left\|y-P_{K} x\right\|^{2}, \quad \forall x \in H, y \in K .
$$

In the context of the variational inequality problem, (2.3) implies that

$$
x^{*} \in \operatorname{VI}(K, A) \Longleftrightarrow x^{*}=P_{K}\left(x^{*}-\lambda A x^{*}\right), \quad \forall \lambda \geq 0 .
$$

If $A$ is an $\alpha$-inverse-strongly monotone mapping of $K$ into $H$, then it is obvious that $A$ is a $(1 / \alpha)$-Lipschitz continuous. We also have that, for all $x, y \in K$ and $r>0$,

$$
\begin{aligned}
\|(I-r A) x-(I-r A) y\|^{2} & =\|x-y-r(A x-A y)\|^{2} \\
& =\|x-y\|^{2}-2 r\langle A x-A y, x-y\rangle+r^{2}\|A x-A y\|^{2} \\
& \leq\|x-y\|^{2}+r(r-2 \alpha)\|A x-A y\|^{2} .
\end{aligned}
$$

So, if $r \leq 2 \alpha$, then $I-r A$ is a nonexpansive mapping of $K$ into $H$.

For solving the generalized mixed equilibrium problem for a bifunction $F: K \times K \rightarrow \mathbb{R}$, let us assume that $F, \varphi$, and $K$ satisfy the following conditions:

(A1) $F(x, x)=0$ for all $x \in K$,

(A2) $F$ is monotone, that is, $F(x, y)+F(y, x) \leq 0$ for all $x, y, \in K$,

(A3) for each $x, y, z \in K, \lim _{t \rightarrow 0} F(t z+(1-t) x, y) \leq F(x, y)$,

(A4) for each $x \in K, y \mapsto F(x, y)$ is convex and lower semicontinuous,

(B1) for each $x \in H$ and $r>0$ there exist a bounded subset $D_{x} \subseteq K$ and $y_{x} \in K$ such that for any $z \in K \backslash D_{x}$,

$$
F\left(z, y_{x}\right)+\varphi\left(y_{x}\right)-\varphi(z)+\frac{1}{r}\left\langle y_{x}-z, z-x\right\rangle<0,
$$

(B2) $K$ is a bounded set.

Then, we have the following lemma. 
Lemma 2.1 (Wangkeeree and Wangkeeree [47]). Assume that $F: K \times K \rightarrow \mathbb{R}$ satisfies (A1)$(A 4)$, and let $\varphi: K \rightarrow \mathbb{R}$ be a proper lower semicontinuous and convex function. Assume that either (B1) or (B2) holds. For $r>0$ and $x \in H$, define a mapping $T_{r}^{(F, \varphi)}: H \rightarrow K$ as follows:

$$
T_{r}^{(F, \varphi)}(x)=\left\{z \in K: F(z, y)+\varphi(y)-\varphi(z)+\frac{1}{r}\langle y-z, z-x\rangle \geq 0, \forall y \in K\right\}
$$

for all $z \in H$. Then, the following hold:

(1) for each $x \in H, T_{r}^{(F, \varphi)} \neq \emptyset$,

(2) $T_{r}^{(F, \varphi)}$ is single-valued,

(3) $T_{r}^{(F, \varphi)}$ is firmly nonexpansive, that is, for any $x, y \in H$,

$$
\left\|T_{r}^{(F, \varphi)} x-T_{r}^{(F, \varphi)} y\right\|^{2} \leq\left\langle T_{r}^{(F, \varphi)} x-T_{r}^{(F, \varphi)} y, x-y\right\rangle,
$$

(4) $F\left(T_{r}^{(F, \varphi)}\right)=\operatorname{GMEP}(F)$,

(5) $\operatorname{GMEP}(F)$ is closed and convex.

We will also use the following lemma in our results.

Lemma 2.2 (Baillon and Haddad [51]). Let E be a Banach space, let $f$ be a continuously Fréchet differentiable convex functional on $E$, and let $\nabla f$ be the gradient of $f$. If $\nabla f$ is $(1 / \alpha)$-Lipschitz continuous, then $\nabla f$ is $\alpha$-inverse-strongly monotone.

\section{Main Results}

Theorem 3.1. Let $K$ be a nonempty closed and convex subset of a real Hilbert space $H$. For each $m=1,2$, let $F_{m}$ be a bifunction from $K \times K$ satisfying $(A 1)-(A 4), \varphi_{m}: K \rightarrow \mathbb{R} \cup\{+\infty\}$ a proper lower semicontinuous and convex function with assumption (B1) or (B2), A be an $\alpha$-inverse-strongly monotone mapping of $K$ into $H$, and $B$ a $\beta$-inverse-strongly monotone mapping of $K$ into $H$, and, for each $i=1,2, \ldots$, let $T_{i}: K \rightarrow K$ be a nonexpansive mapping such that $\bigcap_{i=1}^{\infty} F\left(T_{i}\right) \neq \emptyset$. Let $D$ be a $\gamma$ inverse-strongly monotone mapping of $K$ into $H$. Suppose that $F:=\bigcap_{i=1}^{\infty} F\left(T_{i}\right) \cap \operatorname{GMEP}\left(F_{1}, A, \varphi_{1}\right) \cap$ $\operatorname{GMEP}\left(F_{2}, B, \varphi_{2}\right) \cap \operatorname{VI}(K, D) \neq \emptyset$. Let $\left\{z_{n}\right\}_{n=1}^{\infty},\left\{u_{n}\right\}_{n=1}^{\infty},\left\{w_{n}\right\}_{n=1}^{\infty},\left\{y_{n, i}\right\}_{n=1}^{\infty}(i=1,2, \ldots)$, and $\left\{x_{n}\right\}_{n=0}^{\infty}$ be generated by $x_{0} \in K, C_{1, i}=K, C_{1}=\bigcap_{i=1}^{\infty} C_{1, i}, x_{1}=P_{C_{1}} x_{0}$,

$$
\begin{gathered}
z_{n}=T_{r_{n}}^{\left(F_{1}, \varphi_{1}\right)}\left(x_{n}-r_{n} A x_{n}\right), \\
u_{n}=T_{\lambda_{n}}^{\left(F_{2}, \varphi_{2}\right)}\left(z_{n}-\lambda_{n} B z_{n}\right), \\
w_{n}=P_{K}\left(u_{n}-s_{n} D u_{n}\right), \\
y_{n, i}=\alpha_{n, i} x_{0}+\left(1-\alpha_{n, i}\right) T_{i} w_{n},
\end{gathered}
$$




$$
\begin{gathered}
C_{n+1, i}=\left\{z \in C_{n, i}:\left\|y_{n, i}-z\right\|^{2} \leq\left\|x_{n}-z\right\|^{2}+\alpha_{n, i}\left(\left\|x_{0}\right\|^{2}+2\left\langle x_{n}-x_{0}, z\right\rangle\right)\right\}, \\
C_{n+1}=\bigcap_{i=1}^{\infty} C_{n+1, i} \\
x_{n+1}=P_{C_{n+1}} x_{0}, \quad n \geq 1 .
\end{gathered}
$$

Assume that $\left\{\alpha_{n, i}\right\}_{n=1}^{\infty} \subset(0,1)(i=1,2, \ldots),\left\{r_{n}\right\}_{n=1}^{\infty} \subset[0,2 \alpha]$, and $\left\{\lambda_{n}\right\}_{n=1}^{\infty} \subset[0,2 \beta]$ satisfy

(i) $0<a \leq r_{n} \leq b<2 \alpha$,

(ii) $0<c \leq \lambda_{n} \leq f<2 \beta$,

(iii) $\lim _{n \rightarrow \infty} \alpha_{n, i}=0$,

(iv) $0<h \leq s_{n} \leq j<2 \gamma$.

Then, $\left\{x_{n}\right\}_{n=0}^{\infty}$ converges strongly to $P_{F} x_{0}$.

Proof. Let $x^{*} \in F$. Then,

$$
\begin{aligned}
\left\|w_{n}-x^{*}\right\|^{2} & =\left\|P_{K}\left(u_{n}-s_{n} D u_{n}\right)-P_{K}\left(x^{*}-s_{n} D x^{*}\right)\right\|^{2} \\
& \leq\left\|\left(u_{n}-s_{n} D u_{n}\right)-\left(x^{*}-s_{n} D x^{*}\right)\right\|^{2} \\
& \leq\left\|u_{n}-x^{*}\right\|^{2}+s_{n}\left(s_{n}-2 \gamma\right)\left\|D u_{n}-D x^{*}\right\|^{2} \\
& \leq\left\|u_{n}-x^{*}\right\|^{2} .
\end{aligned}
$$

Since both $I-r_{n} A$ and $I-\lambda_{n} B$ are nonexpansive for each $n \geq 1$ and $x^{*}=T_{r_{n}}^{\left(F_{1}, \varphi_{1}\right)}\left(x^{*}-r_{n} A x^{*}\right)$, $x^{*}=T_{\lambda_{n}}^{\left(F_{2}, \varphi_{2}\right)}\left(x^{*}-\lambda_{n} B x^{*}\right)$, from (2.6), we have that

$$
\begin{aligned}
\left\|u_{n}-x^{*}\right\|^{2} & =\left\|T_{\lambda_{n}}^{\left(F_{2}, \varphi_{2}\right)}\left(z_{n}-\lambda_{n} B z_{n}\right)-x^{*}\right\|^{2} \\
& =\left\|T_{\lambda_{n}}^{\left(F_{2}, \varphi_{2}\right)}\left(z_{n}-\lambda_{n} B z_{n}\right)-T_{\lambda_{n}}^{\left(F_{2}, \varphi_{2}\right)}\left(x^{*}-\lambda_{n} B x^{*}\right)\right\|^{2} \\
& \leq\left\|\left(I-\lambda_{n} B\right) z_{n}-\left(I-\lambda_{n} B\right) x^{*}\right\|^{2} \\
& \leq\left\|z_{n}-x^{*}\right\|^{2}+\lambda_{n}\left(\lambda_{n}-2 \beta\right)\left\|B z_{n}-B x^{*}\right\|^{2} \\
& \leq\left\|z_{n}-x^{*}\right\|^{2} \quad\left(\text { since } \lambda_{n}<2 \beta, \forall n \geq 1\right)
\end{aligned}
$$


International Journal of Mathematics and Mathematical Sciences

$$
\begin{aligned}
\left\|z_{n}-x^{*}\right\|^{2} & =\left\|T_{r_{n}}^{\left(F_{1}, \varphi_{1}\right)}\left(x_{n}-r_{n} A x_{n}\right)-x^{*}\right\|^{2} \\
& =\left\|T_{r_{n}}^{\left(F_{1}, \varphi_{1}\right)}\left(x_{n}-r_{n} A x_{n}\right)-T_{r_{n}}^{\left(F_{1}, \varphi_{1}\right)}\left(x^{*}-r_{n} A x^{*}\right)\right\|^{2} \\
& \leq\left\|\left(I-r_{n} A\right) x_{n}-\left(I-r_{n} A\right) x^{*}\right\|^{2} \\
& \leq\left\|x_{n}-x^{*}\right\|^{2}+r_{n}\left(r_{n}-2 \alpha\right)\left\|A x_{n}-A x^{*}\right\|^{2} \\
& \leq\left\|x_{n}-x^{*}\right\|^{2} .
\end{aligned}
$$

Therefore,

$$
\left\|u_{n}-x^{*}\right\| \leq\left\|x_{n}-x^{*}\right\| .
$$

Let $n=1$, then $C_{1, i}=K$ is closed convex for each $i=1,2, \ldots$ Now assume that $C_{n, i}$ is closed convex for some $n>1$. Then, from definition of $C_{n+1, i}$, we know that $C_{n+1, i}$ is closed convex for the same $n>1$. Hence, $C_{n, i}$ is closed convex for $n \geq 1$ and for each $i=1,2, \ldots$ This implies that $C_{n}$ is closed convex for $n \geq 1$. Furthermore, we show that $F \subset C_{n}$. For $n=1, F \subset K=C_{1, i}$. For $n \geq 2$, let $x^{*} \in F$. Then,

$$
\begin{aligned}
\left\|y_{n, i}-x^{*}\right\|^{2} & =\left\|\alpha_{n, i}\left(x_{0}-x^{*}\right)+\left(1-\alpha_{n, i}\right)\left(T_{i} w_{n}-x^{*}\right)\right\|^{2} \\
& \leq \alpha_{n, i}\left\|x_{0}-x^{*}\right\|^{2}+\left(1-\alpha_{n, i}\right)\left\|w_{n}-x^{*}\right\|^{2} \\
& \leq\left\|w_{n}-x^{*}\right\|^{2}+\alpha_{n, i}\left(\left\|x_{0}-x^{*}\right\|^{2}-\left\|w_{n}-x^{*}\right\|^{2}\right) \\
& \leq\left\|x_{n}-x^{*}\right\|^{2}+\alpha_{n, i}\left(\left\|x_{0}\right\|^{2}+2\left\langle x_{n}-x_{0}, x^{*}\right\rangle\right)
\end{aligned}
$$

which shows that $x^{*} \in C_{n, i}$, for all $n \geq 2$, for all $i=1,2, \ldots$ Thus, $F \subset C_{n, i}$, for all $n \geq 1$, for all $i=1,2, \ldots$. Hence, it follows that $F \subset C_{n}$, for all $n \geq 1$. Since $x_{n}=P_{C_{n}} x_{0}$, for all $n \geq 1$, and $x_{n+1} \in C_{n+1} \subset C_{n}$, for all $n \geq 1$, we have that

$$
\left\|x_{n}-x_{0}\right\| \leq\left\|x_{n+1}-x_{0}\right\|, \quad \forall n \geq 1
$$

Also, as $F \subset C_{n}$ by (2.1), it follows that

$$
\left\|x_{n}-x_{0}\right\| \leq\left\|z-x_{0}\right\|, \quad z \in F, \forall n \geq 1
$$

From (3.6) and (3.7), we have that $\lim _{n \rightarrow \infty}\left\|x_{n}-x_{0}\right\|$ exists. Hence, $\left\{x_{n}\right\}_{n=0}^{\infty}$ is bounded and so are $\left\{z_{n}\right\}_{n=1}^{\infty},\left\{A x_{n}\right\}_{n=1}^{\infty},\left\{u_{n}\right\}_{n=1}^{\infty},\left\{D u_{n}\right\}_{n=1}^{\infty},\left\{B z_{n}\right\}_{n=1}^{\infty},\left\{w_{n}\right\}_{n=1}^{\infty},\left\{T_{i} w_{n}\right\}_{n=1}^{\infty}$, and $\left\{y_{n, i}\right\}_{n=1}^{\infty}, i=$ $1,2, \ldots$. For $m>n \geq 1$, we have that $x_{m}=P_{C_{m}} x_{0} \in C_{m} \subset C_{n}$. By (2.4), we obtain

$$
\left\|x_{m}-x_{n}\right\|^{2} \leq\left\|x_{n}-x_{0}\right\|^{2}-\left\|x_{m}-x_{0}\right\|^{2} \text {. }
$$


Letting $m, n \rightarrow \infty$ and taking the limit in (3.8), we have that $x_{m}-x_{n} \rightarrow 0, m, n \rightarrow \infty$, which shows that $\left\{x_{n}\right\}_{n=0}^{\infty}$ is Cauchy. In particular, $\lim _{n \rightarrow \infty}\left\|x_{n+1}-x_{n}\right\|=0$. Since, $\left\{x_{n}\right\}_{n=0}^{\infty}$ is Cauchy, we assume that $x_{n} \rightarrow z \in K$.

Since $x_{n+1}=P_{C_{n+1}} x_{0} \in C_{n+1}$, then

$$
\left\|y_{n, i}-x_{n+1}\right\|^{2} \leq\left\|x_{n}-x_{n+1}\right\|^{2}+\alpha_{n, i}\left(\left\|x_{0}\right\|^{2}+2\left\langle x_{n}-x_{0}, x_{n+1}\right\rangle\right) \longrightarrow 0,
$$

and it follows that

$$
\left\|y_{n, i}-x_{n}\right\| \leq\left\|y_{n, i}-x_{n+1}\right\|+\left\|x_{n}-x_{n+1}\right\|
$$

Thus,

$$
\lim _{n \rightarrow \infty}\left\|y_{n, i}-x_{n}\right\|=0, \quad i=1,2, \ldots
$$

Furthermore,

$$
\begin{aligned}
\left\|y_{n, i}-x^{*}\right\|^{2} & \leq \alpha_{n, i}\left\|x_{0}-x^{*}\right\|^{2}+\left(1-\alpha_{n, i}\right)\left\|T_{i} w_{n}-x^{*}\right\|^{2} \\
& \leq \alpha_{n, i}\left\|x_{0}-x^{*}\right\|^{2}+\left(1-\alpha_{n, i}\right)\left\|w_{n}-x^{*}\right\|^{2} \\
& \leq \alpha_{n, i}\left\|x_{0}-x^{*}\right\|^{2}+\left(1-\alpha_{n, i}\right)\left\|u_{n}-x^{*}\right\|^{2} \\
& \leq \alpha_{n, i}\left\|x_{0}-x^{*}\right\|^{2}+\left(1-\alpha_{n, i}\right)\left\|T_{\lambda_{n}}^{\left(F_{2}, \varphi_{2}\right)}\left(z_{n}-\lambda_{n} B z_{n}\right)-T_{\lambda_{n}}^{\left(F_{2}, \varphi_{2}\right)}\left(x^{*}-\lambda_{n} B x^{*}\right)\right\|^{2} \\
& \leq \alpha_{n, i}\left\|x_{0}-x^{*}\right\|^{2}+\left(1-\alpha_{n, i}\right)\left\|\left(z_{n}-\lambda_{n} B z_{n}\right)-\left(x^{*}-\lambda_{n} B x^{*}\right)\right\|^{2} \\
& \leq \alpha_{n, i}\left\|x_{0}-x^{*}\right\|^{2}+\left(1-\alpha_{n, i}\right)\left[\left\|z_{n}-x^{*}\right\|^{2}+\lambda_{n}\left(\lambda_{n}-2 \beta\right)\left\|B z_{n}-B x^{*}\right\|^{2}\right] \\
& \leq \alpha_{n, i}\left\|x_{0}-x^{*}\right\|^{2}+\left\|x_{n}-x^{*}\right\|^{2}+\lambda_{n}\left(\lambda_{n}-2 \beta\right)\left\|B z_{n}-B x^{*}\right\|^{2} .
\end{aligned}
$$

Since $0<c \leq \lambda_{n} \leq f<2 \beta$, we have that

$$
\begin{aligned}
c(2 \beta-f)\left\|B z_{n}-B x^{*}\right\|^{2} & \leq\left\|x_{n}-x^{*}\right\|^{2}-\left\|y_{n, i}-x^{*}\right\|^{2}+\alpha_{n, i}\left\|x_{0}-x^{*}\right\|^{2} \\
& \leq\left\|y_{n, i}-x_{n}\right\|\left(\left\|x_{n}-x^{*}\right\|+\left\|y_{n, i}-x^{*}\right\|\right)+\alpha_{n, i}\left\|x_{0}-x^{*}\right\|^{2} .
\end{aligned}
$$

Hence, $\lim _{n \rightarrow \infty}\left\|B z_{n}-B x^{*}\right\|=0$. From (3.1), we have that

$$
\begin{aligned}
\left\|y_{n, i}-x^{*}\right\|^{2} & \leq \alpha_{n, i}\left\|x_{0}-x^{*}\right\|^{2}+\left(1-\alpha_{n, i}\right)\left\|T_{i} w_{n}-x^{*}\right\|^{2} \\
& =\alpha_{n, i}\left\|x_{0}-x^{*}\right\|^{2}+\left(1-\alpha_{n, i}\right)\left\|u_{n}-x^{*}\right\|^{2} \\
& \leq \alpha_{n, i}\left\|x_{0}-x^{*}\right\|^{2}+\left\|u_{n}-x^{*}\right\|^{2} .
\end{aligned}
$$


International Journal of Mathematics and Mathematical Sciences

On the other hand,

$$
\begin{aligned}
\left\|u_{n}-x^{*}\right\|^{2} \leq & \left\|T_{\lambda_{n}}^{\left(F_{2}, \varphi_{2}\right)}\left(z_{n}-\lambda_{n} B z_{n}\right)-T_{\lambda_{n}}^{\left(F_{2}, \varphi_{2}\right)}\left(x^{*}-\lambda_{n} B x^{*}\right)\right\|^{2} \\
\leq & \left\langle\left(z_{n}-\lambda_{n} B z_{n}\right)-\left(x^{*}-\lambda_{n} B x^{*}\right), u_{n}-x^{*}\right\rangle \\
= & \frac{1}{2}\left[\left\|\left(z_{n}-\lambda_{n} B z_{n}\right)-\left(x^{*}-\lambda_{n} B x^{*}\right)\right\|^{2}+\left\|u_{n}-x^{*}\right\|^{2}\right. \\
& \left.\quad-\left\|\left(z_{n}-\lambda_{n} B z_{n}\right)-\left(x^{*}-\lambda_{n} B x^{*}\right)-\left(u_{n}-x^{*}\right)\right\|^{2}\right] \\
\leq & \frac{1}{2}\left[\left\|z_{n}-x^{*}\right\|^{2}+\left\|u_{n}-x^{*}\right\|^{2}-\left\|\left(z_{n}-\lambda_{n} B z_{n}\right)-\left(x^{*}-\lambda_{n} B x^{*}\right)-\left(u_{n}-x^{*}\right)\right\|^{2}\right] \\
= & \frac{1}{2}\left[\left\|z_{n}-x^{*}\right\|^{2}+\left\|u_{n}-x^{*}\right\|^{2}-\left\|u_{n}-z_{n}\right\|^{2}\right. \\
& \left.\quad+2 \lambda_{n}\left\langle z_{n}-u_{n}, B z_{n}-B x^{*}\right\rangle-\lambda_{n}^{2}\left\|B z_{n}-B x^{*}\right\|^{2}\right],
\end{aligned}
$$

and, hence,

$$
\begin{aligned}
\left\|u_{n}-x^{*}\right\|^{2} & \leq\left\|z_{n}-x^{*}\right\|^{2}-\left\|u_{n}-z_{n}\right\|^{2}+2 \lambda_{n}\left\langle z_{n}-u_{n}, B z_{n}-B x^{*}\right\rangle-\lambda_{n}^{2}\left\|B z_{n}-B x^{*}\right\|^{2} \\
& \leq\left\|z_{n}-x^{*}\right\|^{2}-\left\|u_{n}-z_{n}\right\|^{2}+2 \lambda_{n}\left\|z_{n}-u_{n}\right\|\left\|B z_{n}-B x^{*}\right\| .
\end{aligned}
$$

Putting (3.16) into (3.14), we have that

$$
\left\|y_{n, i}-x^{*}\right\|^{2} \leq\left\|z_{n}-x^{*}\right\|^{2}-\left\|u_{n}-z_{n}\right\|^{2}+2 \lambda_{n}\left\|z_{n}-u_{n}\right\|\left\|B z_{n}-B x^{*}\right\|+\alpha_{n, i}\left\|x_{0}-x^{*}\right\|^{2} .
$$

It follows that

$$
\begin{aligned}
\left\|z_{n}-u_{n}\right\|^{2} \leq & \left\|z_{n}-x^{*}\right\|^{2}-\left\|y_{n, i}-x^{*}\right\|^{2}+2 \lambda_{n}\left\|z_{n}-u_{n}\right\|\left\|B z_{n}-B x^{*}\right\|+\alpha_{n, i}\left\|x_{0}-x^{*}\right\|^{2} \\
\leq & \left\|x_{n}-x^{*}\right\|^{2}-\left\|y_{n, i}-x^{*}\right\|^{2}+2 \lambda_{n}\left\|z_{n}-u_{n}\right\|\left\|B z_{n}-B x^{*}\right\|+\alpha_{n, i}\left\|x_{0}-x^{*}\right\|^{2} \\
\leq & \left\|y_{n, i}-x_{n}\right\|\left(\left\|x_{n}-x^{*}\right\|+\left\|y_{n, i}-x^{*}\right\|\right)+2 \lambda_{n}\left\|z_{n}-u_{n}\right\|\left\|B z_{n}-B x^{*}\right\| \\
& +\alpha_{n, i}\left\|x_{0}-x^{*}\right\|^{2} .
\end{aligned}
$$


Therefore, $\lim _{n \rightarrow \infty}\left\|z_{n}-u_{n}\right\|=0$. Furthermore,

$$
\begin{aligned}
\left\|y_{n, i}-x^{*}\right\|^{2} & \leq \alpha_{n, i}\left\|x_{0}-x^{*}\right\|^{2}+\left(1-\alpha_{n, i}\right)\left\|T_{i} w_{n}-x^{*}\right\|^{2} \\
& \leq \alpha_{n, i}\left\|x_{0}-x^{*}\right\|^{2}+\left(1-\alpha_{n, i}\right)\left\|u_{n}-x^{*}\right\|^{2} \\
& \leq \alpha_{n, i}\left\|x_{0}-x^{*}\right\|^{2}+\left(1-\alpha_{n, i}\right)\left\|z_{n}-x^{*}\right\|^{2} \\
& \leq \alpha_{n, i}\left\|x_{0}-x^{*}\right\|^{2}+\left(1-\alpha_{n, i}\right)\left\|T_{r_{n}}^{\left(F_{1}, \varphi_{1}\right)}\left(x_{n}-r_{n} A x_{n}\right)-T_{r_{n}}^{\left(F_{1}, \varphi_{1}\right)}\left(x^{*}-r_{n} A x^{*}\right)\right\|^{2} \\
& \leq \alpha_{n, i}\left\|x_{0}-x^{*}\right\|^{2}+\left(1-\alpha_{n, i}\right)\left\|\left(x_{n}-r_{n} A x_{n}\right)-\left(x^{*}-r_{n} A x^{*}\right)\right\|^{2} \\
& \leq \alpha_{n, i}\left\|x_{0}-x^{*}\right\|^{2}+\left(1-\alpha_{n, i}\right)\left[\left\|x_{n}-x^{*}\right\|^{2}+r_{n}\left(r_{n}-2 \alpha\right)\left\|A x_{n}-A x^{*}\right\|^{2}\right] \\
& \leq \alpha_{n, i}\left\|x_{0}-x^{*}\right\|^{2}+\left\|x_{n}-x^{*}\right\|^{2}+r_{n}\left(r_{n}-2 \alpha\right)\left\|A x_{n}-A x^{*}\right\|^{2} .
\end{aligned}
$$

Since $0<a \leq r_{n} \leq b<2 \alpha$, we have that

$$
\begin{aligned}
a(2 \alpha-b)\left\|A x_{n}-A x^{*}\right\|^{2} & \leq\left\|x_{n}-x^{*}\right\|^{2}-\left\|y_{n, i}-x^{*}\right\|^{2}+\alpha_{n, i}\left\|x_{0}-x^{*}\right\|^{2} \\
& \leq\left\|y_{n, i}-x_{n}\right\|\left(\left\|x_{n}-x^{*}\right\|+\left\|y_{n, i}-x^{*}\right\|\right)+\alpha_{n, i}\left\|x_{0}-x^{*}\right\|^{2} .
\end{aligned}
$$

Hence, $\lim _{n \rightarrow \infty}\left\|A x_{n}-A x^{*}\right\|=0$. From (3.1), we have that

$$
\begin{aligned}
\left\|y_{n, i}-x^{*}\right\|^{2} & \leq \alpha_{n, i}\left\|x_{0}-x^{*}\right\|^{2}+\left(1-\alpha_{n, i}\right)\left\|T_{i} w_{n}-x^{*}\right\|^{2} \\
& \leq \alpha_{n, i}\left\|x_{0}-x^{*}\right\|^{2}+\left(1-\alpha_{n, i}\right)\left\|u_{n}-x^{*}\right\|^{2} \\
& \leq \alpha_{n, i}\left\|x_{0}-x^{*}\right\|^{2}+\left\|z_{n}-x^{*}\right\|^{2} .
\end{aligned}
$$

On the other hand,

$$
\begin{aligned}
\left\|z_{n}-x^{*}\right\|^{2} \leq & \left\|T_{r_{n}}^{\left(F_{1}, \varphi_{1}\right)}\left(x_{n}-r_{n} A x_{n}\right)-T_{r_{n}}^{\left(F_{1}, \varphi_{1}\right)}\left(x^{*}-r_{n} A x^{*}\right)\right\|^{2} \\
\leq & \left\langle\left(x_{n}-r_{n} A x_{n}\right)-\left(x^{*}-r_{n} A x^{*}\right), z_{n}-x^{*}\right\rangle \\
= & \frac{1}{2}\left[\left\|\left(x_{n}-r_{n} A x_{n}\right)-\left(x^{*}-r_{n} A x^{*}\right)\right\|^{2}+\left\|z_{n}-x^{*}\right\|^{2}\right. \\
& \left.\quad-\left\|\left(x_{n}-r_{n} A x_{n}\right)-\left(x^{*}-r_{n} A x^{*}\right)-\left(z_{n}-x^{*}\right)\right\|^{2}\right] \\
\leq & \frac{1}{2}\left[\left\|x_{n}-x^{*}\right\|^{2}+\left\|z_{n}-x^{*}\right\|^{2}-\left\|\left(x_{n}-r_{n} A x_{n}\right)-\left(x^{*}-r_{n} A x^{*}\right)-\left(z_{n}-x^{*}\right)\right\|^{2}\right] \\
=\frac{1}{2}\left[\left\|x_{n}-x^{*}\right\|^{2}+\left\|z_{n}-x^{*}\right\|^{2}-\left\|z_{n}-x_{n}\right\|^{2}\right. & \left.\quad+2 r_{n}\left\langle x_{n}-z_{n}, A x_{n}-A x^{*}\right\rangle-r_{n}^{2}\left\|A x_{n}-A x^{*}\right\|^{2}\right],
\end{aligned}
$$


and, hence,

$$
\begin{aligned}
\left\|z_{n}-x^{*}\right\|^{2} & \leq\left\|x_{n}-x^{*}\right\|^{2}-\left\|z_{n}-x_{n}\right\|^{2}+2 r_{n}\left\langle x_{n}-z_{n}, A x_{n}-A x^{*}\right\rangle-r_{n}^{2}\left\|A x_{n}-A x^{*}\right\|^{2} \\
& \leq\left\|x_{n}-x^{*}\right\|^{2}-\left\|z_{n}-x_{n}\right\|^{2}+2 r_{n}\left\|x_{n}-z_{n}\right\|\left\|A x_{n}-A x^{*}\right\| .
\end{aligned}
$$

Putting (3.23) into (3.21), we have that

$$
\left\|y_{n, i}-x^{*}\right\|^{2} \leq\left\|x_{n}-x^{*}\right\|^{2}-\left\|z_{n}-x_{n}\right\|^{2}+2 r_{n}\left\|x_{n}-z_{n}\right\|\left\|A x_{n}-A x^{*}\right\|+\alpha_{n, i}\left\|x_{0}-x^{*}\right\|^{2} .
$$

It follows that

$$
\begin{aligned}
\left\|x_{n}-z_{n}\right\|^{2} & \leq\left\|x_{n}-x^{*}\right\|^{2}-\left\|y_{n, i}-x^{*}\right\|^{2}+2 r_{n}\left\|x_{n}-z_{n}\right\|\left\|A x_{n}-A x^{*}\right\|+\alpha_{n, i}\left\|x_{0}-x^{*}\right\|^{2} \\
& \leq\left\|y_{n, i}-x_{n}\right\|\left(\left\|x_{n}-x^{*}\right\|+\left\|y_{n, i}-x^{*}\right\|\right)+2 r_{n}\left\|x_{n}-z_{n}\right\|\left\|A x_{n}-A x^{*}\right\|+\alpha_{n, i}\left\|x_{0}-x^{*}\right\|^{2} .
\end{aligned}
$$

Therefore, $\lim _{n \rightarrow \infty}\left\|x_{n}-z_{n}\right\|=0$. But $y_{n, i}=\alpha_{n, i} x_{0}+\left(1-\alpha_{n, i}\right) T_{i} w_{n}$ implies that

$$
\left\|y_{n, i}-T_{i} w_{n}\right\|=\alpha_{n, i}\left\|x_{0}-T_{i} w_{n}\right\| \longrightarrow 0
$$

Furthermore, we have that

$$
\left\|x_{n}-T_{i} w_{n}\right\| \leq\left\|y_{n, i}-T_{i} w_{n}\right\|+\left\|y_{n, i}-x_{n}\right\| \longrightarrow 0
$$

Furthermore,

$$
\begin{aligned}
\left\|y_{n, i}-x^{*}\right\|^{2} & \leq \alpha_{n, i}\left\|x_{0}-x^{*}\right\|^{2}+\left(1-\alpha_{n, i}\right)\left\|T_{i} w_{n}-x^{*}\right\|^{2} \\
& \leq \alpha_{n, i}\left\|x_{0}-x^{*}\right\|^{2}+\left(1-\alpha_{n, i}\right)\left\|w_{n}-x^{*}\right\|^{2} \\
& \leq \alpha_{n, i}\left\|x_{0}-x^{*}\right\|^{2}+\left(1-\alpha_{n, i}\right)\left\|P_{K}\left(u_{n}-s_{n} D u_{n}\right)-P_{K}\left(x^{*}-s_{n} D x^{*}\right)\right\|^{2} \\
& \leq \alpha_{n, i}\left\|x_{0}-x^{*}\right\|^{2}+\left(1-\alpha_{n, i}\right)\left\|\left(u_{n}-s_{n} D u_{n}\right)-\left(x^{*}-s_{n} D x^{*}\right)\right\|^{2} \\
& \leq \alpha_{n, i}\left\|x_{0}-x^{*}\right\|^{2}+\left(1-\alpha_{n, i}\right)\left[\left\|u_{n}-x^{*}\right\|^{2}+s_{n}\left(s_{n}-2 \gamma\right)\left\|D u_{n}-D x^{*}\right\|^{2}\right] \\
& \leq \alpha_{n, i}\left\|x_{0}-x^{*}\right\|^{2}+\left\|x_{n}-x^{*}\right\|^{2}+\left(1-\alpha_{n, i}\right) s_{n}\left(s_{n}-2 \gamma\right)\left\|D u_{n}-D x^{*}\right\|^{2} .
\end{aligned}
$$

Thus,

$$
\begin{aligned}
\left(1-d_{i}\right) h(2 \gamma-j)\left\|D u_{n}-D x^{*}\right\|^{2} & \leq\left(1-\alpha_{n, i}\right) s_{n}\left(2 \gamma-s_{n}\right)\left\|D u_{n}-D x^{*}\right\|^{2} \\
& \leq \alpha_{n, i}\left\|x_{0}-x^{*}\right\|^{2}+\left\|x_{n}-x^{*}\right\|^{2}-\left\|y_{n, i}-x^{*}\right\|^{2} \\
& \leq \alpha_{n, i}\left\|x_{0}-x^{*}\right\|^{2}+\left\|y_{n, i}-x_{n}\right\|\left(\left\|x_{n}-x^{*}\right\|+\left\|y_{n, i}-x^{*}\right\|\right)
\end{aligned}
$$


Since $0<h \leq s_{n} \leq j<2 \gamma$, condition (iii) and $\left\|y_{n, i}-x_{n}\right\| \rightarrow 0$ as $n \rightarrow \infty$, we have that $\lim _{n \rightarrow \infty}\left\|D u_{n}-D x^{*}\right\|=0$. Now, using (2.2), we obtain

$$
\begin{aligned}
\left\|w_{n}-x^{*}\right\|^{2} \leq & \left\|P_{K}\left(u_{n}-s_{n} D u_{n}\right)-P_{K}\left(x^{*}-s_{n} D x^{*}\right)\right\|^{2} \\
\leq & \left\langle\left(u_{n}-s_{n} D u_{n}\right)-\left(x^{*}-s_{n} D x^{*}\right), w_{n}-x^{*}\right\rangle \\
= & \frac{1}{2}\left[\left\|\left(u_{n}-s_{n} D u_{n}\right)-\left(x^{*}-s_{n} D x^{*}\right)\right\|^{2}+\left\|w_{n}-x^{*}\right\|^{2}\right. \\
& \left.\quad-\left\|\left(u_{n}-s_{n} D u_{n}\right)-\left(x^{*}-s_{n} D x^{*}\right)-\left(w_{n}-x^{*}\right)\right\|^{2}\right] \\
\leq & \frac{1}{2}\left[\left\|u_{n}-x^{*}\right\|^{2}+\left\|w_{n}-x^{*}\right\|^{2}-\left\|\left(u_{n}-s_{n} D u_{n}\right)-\left(x^{*}-s_{n} D x^{*}\right)-\left(w_{n}-x^{*}\right)\right\|^{2}\right] \\
= & \frac{1}{2}\left[\left\|x_{n}-x^{*}\right\|^{2}+\left\|w_{n}-x^{*}\right\|^{2}-\left\|w_{n}-u_{n}\right\|^{2}+2 s_{n}\left\langle u_{n}-w_{n}, D u_{n}-D x^{*}\right\rangle\right. \\
& \left.\quad-s_{n}^{2}\left\|D u_{n}-D x^{*}\right\|^{2}\right] .
\end{aligned}
$$

Thus,

$$
\left\|w_{n}-x^{*}\right\|^{2} \leq\left\|x_{n}-x^{*}\right\|^{2}-\left\|w_{n}-u_{n}\right\|^{2}+2 s_{n}\left\|w_{n}-u_{n}\right\|\left\|D u_{n}-D x^{*}\right\| .
$$

Using this last inequality, we obtain from (3.1)

$$
\begin{aligned}
\left\|y_{n, i}-x^{*}\right\|^{2} \leq & \alpha_{n, i}\left\|x_{0}-x^{*}\right\|^{2}+\left(1-\alpha_{n, i}\right)\left\|T_{i} w_{n}-x^{*}\right\|^{2} \\
\leq & \alpha_{n, i}\left\|x_{0}-x^{*}\right\|^{2}+\left(1-\alpha_{n, i}\right)\left\|w_{n}-x^{*}\right\|^{2} \\
\leq & \alpha_{n, i}\left\|x_{0}-x^{*}\right\|^{2}+\left\|x_{n}-x^{*}\right\|^{2}-\left(1-\alpha_{n, i}\right)\left\|w_{n}-u_{n}\right\|^{2} \\
& +2 s_{n}\left(1-\alpha_{n, i}\right)\left\|w_{n}-u_{n}\right\|\left\|D u_{n}-\mathrm{D} x^{*}\right\| .
\end{aligned}
$$

This implies that

$$
\begin{aligned}
\left(1-\alpha_{n, i}\right)\left\|w_{n}-u_{n}\right\|^{2} \leq & \alpha_{n, i}\left\|x_{0}-x^{*}\right\|^{2}+\left\|x_{n}-x^{*}\right\|^{2}-\left\|y_{n, i}-x^{*}\right\|^{2} \\
& +2 s_{n}\left(1-\alpha_{n, i}\right)\left\|w_{n}-u_{n}\right\|\left\|D u_{n}-D x^{*}\right\| \\
\leq & \alpha_{n, i}\left\|x_{0}-x^{*}\right\|^{2}+\left\|y_{n, i}-x_{n}\right\|\left(\left\|x_{n}-x^{*}\right\|+\left\|y_{n, i}-x^{*}\right\|\right) \\
& +2 s_{n}\left(1-\alpha_{n, i}\right)\left\|w_{n}-u_{n}\right\|\left\|D u_{n}-D x^{*}\right\| .
\end{aligned}
$$

Since $\lim _{n \rightarrow \infty} \alpha_{n, i}=0,\left\|y_{n, i}-x_{n}\right\| \rightarrow 0$ as $n \rightarrow \infty$, and $\left\|D u_{n}-D x^{*}\right\| \rightarrow 0$ as $n \rightarrow \infty$, we have that $\lim _{n \rightarrow \infty}\left\|w_{n}-u_{n}\right\|=0$. Also since $\lim _{n \rightarrow \infty}\left\|w_{n}-x_{n}\right\|=0$ and $\lim _{n \rightarrow \infty}\left\|x_{n}-z\right\|=0$, we have that $\lim _{n \rightarrow \infty}\left\|w_{n}-z\right\|=0$. Now,

$$
\left\|w_{n}-T_{i} w_{n}\right\| \leq\left\|x_{n}-T_{i} w_{n}\right\|+\left\|w_{n}-x_{n}\right\| .
$$


Hence, $\lim _{n \rightarrow \infty}\left\|w_{n}-T_{i} w_{n}\right\|=0, i=1,2, \ldots$ By $\lim _{n \rightarrow \infty}\left\|w_{n}-z\right\|=0$ and $\lim _{n \rightarrow \infty}\left\|w_{n}-T_{i} w_{n}\right\|=$ $0, i=1,2, \ldots$, we have that $z \in \bigcap_{i=1}^{\infty} F\left(T_{i}\right)$.

Since $z_{n}:=T_{r_{n}}^{\left(F_{1}, \varphi_{1}\right)}\left(x_{n}-r_{n} A x_{n}\right), n \geq 1$, we have, for any $y \in K$, that

$$
F_{1}\left(z_{n}, y\right)+\varphi_{1}(y)-\varphi_{1}\left(z_{n}\right)+\left\langle A x_{n}, y-z_{n}\right\rangle+\frac{1}{r_{n}}\left\langle y-z_{n}, z_{n}-x_{n}\right\rangle \geq 0
$$

Furthermore, replacing $n$ by $n_{j}$ in the last inequality and using (A2), we obtain

$$
\varphi_{1}(y)-\varphi_{1}\left(z_{n_{j}}\right)+\left\langle A x_{n_{j}}, y-z_{n_{j}}\right\rangle+\frac{1}{r_{n_{j}}}\left\langle y-z_{n_{j}}, z_{n_{j}}-x_{n_{j}}\right\rangle \geq F_{1}\left(y, z_{n_{j}}\right) .
$$

Let $z_{t}:=t y+(1-t) z$ for all $t \in(0,1]$ and $y \in K$. This implies that $z_{t} \in K$. Then, we have that

$$
\begin{aligned}
\left\langle z_{t}-z_{n_{j}}, A z_{t}\right\rangle \geq & \varphi_{1}\left(z_{n_{j}}\right)-\varphi_{1}\left(z_{t}\right)+\left\langle z_{t}-z_{n_{j}}, A z_{t}\right\rangle-\left\langle z_{t}-z_{n_{j}}, A x_{n_{j}}\right\rangle \\
& -\left\langle z_{t}-z_{n_{j}}, \frac{z_{n_{j}}-x_{n_{j}}}{r_{n_{j}}}\right\rangle+F_{1}\left(z_{t}, z_{n_{j}}\right) \\
= & \varphi_{1}\left(z_{n_{j}}\right)-\varphi_{1}\left(z_{t}\right)+\left\langle z_{t}-z_{n_{j}}, A z_{t}-A z_{n_{j}}\right\rangle \\
& +\left\langle z_{t}-z_{n_{j}}, A z_{n_{j}}-A x_{n_{j}}\right\rangle-\left\langle z_{t}-z_{n_{j}}, \frac{z_{n_{j}}-x_{n_{j}}}{r_{n_{j}}}\right\rangle+F_{1}\left(z_{t}, z_{n_{j}}\right) .
\end{aligned}
$$

Since $\left\|x_{n_{j}}-z_{n_{j}}\right\| \rightarrow 0, j \rightarrow \infty$, we obtain $\left\|A x_{n_{j}}-A z_{n_{j}}\right\| \rightarrow 0, j \rightarrow \infty$. Furthermore, by the monotonicity of $A$, we obtain $\left\langle z_{t}-z_{n_{j}}, A z_{t}-A z_{n_{j}}\right\rangle \geq 0$. Then, by (A4), we obtain (noting that $\left.z_{n_{j}} \rightarrow z\right)$

$$
\left\langle z_{t}-z, A z_{t}\right\rangle \geq \varphi_{1}(z)-\varphi_{1}\left(z_{t}\right)+F_{1}\left(z_{t}, z\right), \quad j \longrightarrow \infty
$$

Using (A1), (A4), and (3.38), we also obtain

$$
\begin{aligned}
0 & =F_{1}\left(z_{t}, z_{t}\right)+\varphi_{1}\left(z_{t}\right)-\varphi_{1}\left(z_{t}\right) \\
& \leq t F_{1}\left(z_{t}, y\right)+(1-t) F_{1}\left(z_{t}, z\right)+t \varphi_{1}(y)+(1-t) \varphi_{1}(z)-\varphi_{1}\left(z_{t}\right) \\
& \leq t\left[F_{1}\left(z_{t}, y\right)+\varphi_{1}(y)-\varphi_{1}\left(z_{t}\right)\right]+(1-t)\left\langle z_{t}-z, A z_{t}\right\rangle \\
& =t\left[F_{1}\left(z_{t}, y\right)+\varphi_{1}(y)-\varphi_{1}\left(z_{t}\right)\right]+(1-t) t\left\langle y-z, A z_{t}\right\rangle,
\end{aligned}
$$

and, hence,

$$
0 \leq F_{1}\left(z_{t}, y\right)+\varphi_{1}(y)-\varphi_{1}\left(z_{t}\right)+(1-t)\left\langle y-z, A z_{t}\right\rangle
$$


Letting $t \rightarrow 0$, we have, for each $y \in K$, that

$$
0 \leq F_{1}(z, y)+\varphi_{1}(y)-\varphi_{1}(z)+\langle y-z, A z\rangle \text {. }
$$

This implies that $z \in \operatorname{GMEP}\left(F_{1}, A, \varphi_{1}\right)$. By following the same arguments, we can show that $z \in \operatorname{GMEP}\left(F_{2}, B, \varphi_{2}\right)$.

Following the arguments of [3, Theorem 3.1, pages 346-347], we can show that $z \in$ $\mathrm{VI}(K, D)$. Therefore, $z \in \bigcap_{i=1}^{\infty} F\left(T_{i}\right) \cap \operatorname{GMEP}\left(F_{1}, A, \varphi_{1}\right) \cap \operatorname{GMEP}\left(F_{2}, B, \varphi_{2}\right) \cap \operatorname{VI}(K, D)$.

Noting that $x_{n}=P_{C_{n}} x_{0}$, we have by (2.3),

$$
\left\langle x_{0}-x_{n}, y-x_{n}\right\rangle \leq 0
$$

for all $y \in C_{n}$. Since $F \subset C_{n}$ and by the continuity of inner product, we obtain, from the above inequality,

$$
\left\langle x_{0}-z, y-z\right\rangle \leq 0,
$$

for all $y \in F$. By (2.3) again, we conclude that $z=P_{F} x_{0}$. This completes the proof.

Corollary 3.2. Let $K$ be a nonempty closed and convex subset of a real Hilbert space $H$. Let $T: K \rightarrow$ $K$ be a nonexpansive mapping such that $F(T) \neq \emptyset$. Let $\left\{x_{n}\right\}_{n=0}^{\infty}$ be generated by

$$
\begin{gathered}
x_{0} \in K, \\
y_{n}=\alpha_{n} x_{0}+\left(1-\alpha_{n}\right) T x_{n}, \\
C_{n+1}=\left\{z \in C_{n}:\left\|y_{n}-z\right\|^{2} \leq\left\|x_{n}-z\right\|^{2}+\alpha_{n}\left(\left\|x_{0}\right\|^{2}+2\left\langle x_{n}-x_{0}, z\right\rangle\right)\right\}, \\
x_{n+1}=P_{C_{n+1}} x_{0}, \quad n \geq 1 .
\end{gathered}
$$

Assume that $\left\{\alpha_{n}\right\}_{n=1}^{\infty} \subset(0,1)$ such that $\lim _{n \rightarrow \infty} \alpha_{n}=0$. Then, $\left\{x_{n}\right\}_{n=0}^{\infty}$ converges strongly to $P_{F(T)} x_{0}$.

Remark 3.3. Corollary 3.2 can be viewed as an improvement of Theorem 3.1 of Martinez-Yanes and $\mathrm{Xu}$ [39] because we relax the iterative step $\mathrm{Q}_{n}$ in the algorithm of Theorem 3.1 of [39].

\section{Applications}

Let $C$ be a nonempty closed and convex cone in $H$ and $D$ an operator of $C$ into $H$. We define the polar of $C$ in $H$ to be the set

$$
K^{*}:=\left\{y^{*} \in H:\left\langle x, y^{*}\right\rangle \geq 0, \forall x \in C\right\}
$$

Then, the element $u \in C$ is called a solution of the complementarity problem if

$$
D u \in K^{*},\langle u, D u\rangle=0 .
$$


The set of solutions of the complementarity problem is denoted by $C(C, D)$. We will assume that $D$ satisfies the following conditions:

(E1) $D$ is $\gamma$-inverse strongly monotone,

(E2) $C(C, D) \neq \emptyset$.

Also, we replace conditions (B1) and (B2) with

(D1) for each $x \in H$ and $r>0$, there exist a bounded subset $D_{x} \subseteq C$ and $y_{x} \in C$ such that, for any $z \in C \backslash D_{x}$,

$$
F\left(z, y_{x}\right)+\varphi\left(y_{x}\right)-\varphi(z)+\frac{1}{r}\left\langle y_{x}-z, z-x\right\rangle<0
$$

(D2) $C$ is a bounded set.

Theorem 4.1. Let $C$ be a nonempty closed and convex subset of a real Hilbert space H. For each $m=1,2$, let $F_{m}$ be a bifunction from $C \times C$ satisfying $(A 1)-(A 4), \varphi_{m}: C \rightarrow \mathbb{R} \cup\{+\infty\}$ a proper lower semicontinuous and convex function with assumption (B1) or (B2), $A$ an $\alpha$-inverse-strongly monotone mapping of $C$ into $H$, and $B$ a $\beta$-inverse-strongly monotone mapping of $C$ into $H$, and, for each $i=1,2, \ldots$, let $T_{i}: C \rightarrow C$ be a nonexpansive mapping such that $\bigcap_{i=1}^{\infty} F\left(T_{i}\right) \neq \emptyset$. Let $D$ be a $\gamma$ inverse-strongly monotone mapping of $K$ into $H$. Suppose that $F:=\bigcap_{i=1}^{\infty} F\left(T_{i}\right) \cap \operatorname{GMEP}\left(F_{1}, A, \varphi_{1}\right) \cap$ $\operatorname{GMEP}\left(F_{2}, B, \varphi_{2}\right) \cap C(C, D) \neq \emptyset$. Let $\left\{z_{n}\right\}_{n=1}^{\infty},\left\{u_{n}\right\}_{n=1}^{\infty},\left\{w_{n}\right\}_{n=1}^{\infty},\left\{y_{n, i}\right\}_{n=1}^{\infty}(i=1,2, \ldots)$, and $\left\{x_{n}\right\}_{n=0}^{\infty}$ be generated by $x_{0} \in C, C_{1, i}=C, C_{1}=\bigcap_{i=1}^{\infty} C_{1, i}, x_{1}=P_{C_{1}} x_{0}$,

$$
\begin{gathered}
z_{n}=T_{r_{n}}^{\left(F_{1}, \varphi_{1}\right)}\left(x_{n}-r_{n} A x_{n}\right), \\
u_{n}=T_{\lambda_{n}}^{\left(F_{2}, \varphi_{2}\right)}\left(z_{n}-\lambda_{n} B z_{n}\right), \\
w_{n}=P_{C}\left(u_{n}-s_{n} D u_{n}\right), \\
y_{n, i}=\alpha_{n, i} x_{0}+\left(1-\alpha_{n, i}\right) T_{i} w_{n}, \\
C_{n+1, i}=\left\{z \in C_{n, i}:\left\|y_{n, i}-z\right\|^{2} \leq\left\|x_{n}-z\right\|^{2}+\alpha_{n, i}\left(\left\|x_{0}\right\|^{2}+2\left\langle x_{n}-x_{0}, z\right\rangle\right)\right\}, \\
C_{n+1}=\bigcap_{i=1}^{\infty} C_{n+1, i} \\
x_{n+1}=P_{C_{n+1}} x_{0}, \quad n \geq 1 .
\end{gathered}
$$

Assume that $\left\{\alpha_{n, i}\right\}_{n=1}^{\infty} \subset(0,1)(i=1,2, \ldots),\left\{r_{n}\right\}_{n=1}^{\infty} \subset[0,2 \alpha]$, and $\left\{\lambda_{n}\right\}_{n=1}^{\infty} \subset[0,2 \beta]$ satisfy

(i) $0<a \leq r_{n} \leq b<2 \alpha$,

(ii) $0<c \leq \lambda_{n} \leq f<2 \beta$,

(iii) $\lim _{n \rightarrow \infty} \alpha_{n, i}=0$,

(iv) $0<h \leq s_{n} \leq j<2 \gamma$.

Then, $\left\{x_{n}\right\}_{n=0}^{\infty}$ converges strongly to $P_{F} x_{0}$. 
Proof. Using Lemma 7.1.1 of [52], we have that $\operatorname{VI}(C, D)=C(C, D)$. Hence, by Theorem 3.1 we obtain the desired conclusion.

Next we study the problem of finding a minimizer of a continuously Fréchet differentiable convex functional in a Hilbert space.

Theorem 4.2. For each $m=1,2$, let $F_{m}$ be a bifunction from $H \times H$ satisfying (A1)-(A4), $\varphi_{m}$ : $H \rightarrow \mathbb{R} \cup\{+\infty\}$ a proper lower semicontinuous and convex function with assumption (B1) or (B2), $A$ an $\alpha$-inverse-strongly monotone mapping of $H$ into itself, and $B$ a $\beta$-inverse-strongly monotone mapping of $H$ into itself, and, for each $i=1,2, \ldots$, let $T_{i}: H \rightarrow H$ be a nonexpansive mapping such that $\bigcap_{i=1}^{\infty} F\left(T_{i}\right) \neq \emptyset$. Suppose that $f$ is a functional on $H$ which satisfies the following conditions:

(1) $f$ is a continuously Fréchet differentiable convex functional on $H$ and $\nabla f$ is $(1 / \gamma)$ Lipschitz continuous,

(2) $(\nabla f)^{-1} 0=\left\{z \in H: f(z)=\min _{y \in H} f(y)\right\} \neq \emptyset$.

Suppose that $F:=\bigcap_{i=1}^{\infty} F\left(T_{i}\right) \cap \operatorname{GMEP}\left(F_{1}, A, \varphi_{1}\right) \cap \operatorname{GMEP}\left(F_{2}, B, \varphi_{2}\right) \cap(\nabla f)^{-1} 0 \neq \emptyset$. Let $\left\{z_{n}\right\}_{n=1}^{\infty}$, $\left\{u_{n}\right\}_{n=1}^{\infty},\left\{w_{n}\right\}_{n=1}^{\infty},\left\{y_{n, i}\right\}_{n=1}^{\infty}(i=1,2, \ldots)$, and $\left\{x_{n}\right\}_{n=0}^{\infty}$ be generated by $x_{0} \in K, C_{1, i}=K, C_{1}=$ $\bigcap_{i=1}^{\infty} C_{1, i}, x_{1}=P_{C_{1}} x_{0}$,

$$
\begin{gathered}
z_{n}=T_{r_{n}}^{\left(F_{1}, \varphi_{1}\right)}\left(x_{n}-r_{n} A x_{n}\right), \\
u_{n}=T_{\left.\lambda_{n}, \varphi_{2}\right)}^{\left(F_{2}\right)}\left(z_{n}-\lambda_{n} B z_{n}\right), \\
w_{n}=u_{n}-s_{n} \nabla f\left(u_{n}\right), \\
y_{n, i}=\alpha_{n, i} w_{n}+\left(1-\alpha_{n, i}\right) T_{i} w_{n}, \\
C_{n+1, i}=\left\{z \in C_{n, i}:\left\|y_{n, i}-z\right\|^{2} \leq\left\|x_{n}-z\right\|^{2}+\alpha_{n, i}\left(\left\|x_{0}\right\|^{2}+2\left\langle x_{n}-x_{0}, z\right\rangle\right)\right\}, \\
C_{n+1}=\bigcap_{i=1}^{\infty} C_{n+1, i}, \\
x_{n+1}=P_{C_{n+1}} x_{0}, \quad n \geq 1 .
\end{gathered}
$$

Assume that $\left\{\alpha_{n, i}\right\}_{n=1}^{\infty} \subset(0,1)(i=1,2, \ldots),\left\{r_{n}\right\}_{n=1}^{\infty} \subset[0,2 \alpha]$, and $\left\{\lambda_{n}\right\}_{n=1}^{\infty} \subset[0,2 \beta]$ satisfy

(i) $0<a \leq r_{n} \leq b<2 \alpha$,

(ii) $0<c \leq \lambda_{n} \leq f<2 \beta$,

(iii) $\lim _{n \rightarrow \infty} \alpha_{n, i}=0$,

(iv) $0<h \leq s_{n} \leq j<2 \gamma$.

Then, $\left\{x_{n}\right\}_{n=0}^{\infty}$ converges strongly to $P_{F} x_{0}$.

Proof. We know from condition (i) and Lemma 2.2 that $\nabla f$ is an $\gamma$-inverse-strongly monotone operator from $H$ into $H$. Using Theorem 3.1, we have the desired conclusion. 

solutions:

We now study a kind of multiobjective optimization problem with nonempty set of

$$
\min h_{1}(x), \quad \min h_{2}(x), \quad x \in K,
$$

where $K$ is a nonempty closed convex subset of a real Hilbert space $H$, and $h_{i}: K \rightarrow \mathbb{R}, i=$ 1,2 , is a convex and a lower semicontinuous functional. Let us denote the set of solutions to (4.6) by $\Omega$ and assume that $\Omega \neq \emptyset$.

We will denote the set of solutions of the following two optimization problems by $\Omega_{1}$ and $\Omega_{2}$, respectively:

$$
\min _{x \in K} h_{1}(x), \quad \min _{x \in K} h_{2}(x)
$$

Clearly, if we find a solution $x \in \Omega_{1} \cap \Omega_{2}$, then one must have $x \in \Omega$.

Now, for each $i=1,2$, let $F_{i}: K \times K \rightarrow \mathbb{R}$ be defined by $F_{i}(x, y):=h_{i}(y)-h_{i}(x)$. Let us now find the following equilibrium problem: find $x \in K$ such that

$$
F_{i}(x, y) \geq 0, \quad i=1,2,
$$

for all $y \in K$. It is obvious that $F_{i}$ satisfies conditions (A1)-(A4) and $\operatorname{EP}\left(F_{i}\right)=\Omega_{i}, i=1,2$, where $\operatorname{EP}\left(F_{i}\right)$ is the set of solutions to (4.8). By Theorem 3.1, we have the following theorem.

Theorem 4.3. Let $K$ be a nonempty closed and convex subset of a real Hilbert space $H$. For each $i=1,2$, let $h_{i}$ be a lower semicontinuous and convex function such that $\Omega_{1} \cap \Omega_{2} \neq \emptyset$. Let $\left\{z_{n}\right\}_{n=1}^{\infty}$, $\left\{u_{n}\right\}_{n=1}^{\infty},\left\{y_{n}\right\}_{n=1}^{\infty}$, and $\left\{x_{n}\right\}_{n=0}^{\infty}$ be generated by $x_{0} \in K, C_{1}=K, x_{1}=P_{C_{1}} x_{0}$,

$$
\begin{gathered}
h_{1}(y)-h_{1}\left(z_{n}\right)+\frac{1}{r_{n}}\left\langle y-z_{n}, z_{n}-x_{n}\right\rangle \geq 0, \quad \forall y \in K, \\
h(y)-h\left(u_{n}\right)+\frac{1}{\lambda_{n}}\left\langle y-u_{n}, u_{n}-z_{n}\right\rangle \geq 0, \quad \forall y \in K, \\
y_{n}=\alpha_{n} x_{0}+\left(1-\alpha_{n}\right) u_{n}, \\
C_{n+1}=\left\{z \in C_{n}:\left\|y_{n}-z\right\|^{2} \leq\left\|x_{n}-z\right\|^{2}+\alpha_{n}\left(\left\|x_{0}\right\|^{2}+2\left\langle x_{n}-x_{0}, z\right\rangle\right)\right\}, \\
x_{n+1}=P_{C_{n+1}} x_{0}, \quad n \geq 1 .
\end{gathered}
$$

Assume that $\left\{\alpha_{n}\right\}_{n=1}^{\infty} \subset(0,1),\left\{r_{n}\right\}_{n=1}^{\infty} \subset(0, \infty)$, and $\left\{\lambda_{n}\right\}_{n=1}^{\infty} \subset(0, \infty)$ satisfy

(i) $\liminf \operatorname{in}_{n \rightarrow \infty} r_{n}>0$,

(ii) $\liminf _{n \rightarrow \infty} \lambda_{n}>0$,

(iii) $\lim _{n \rightarrow \infty} \alpha_{n}=0$.

Then, $\left\{x_{n}\right\}_{n=0}^{\infty}$ converges strongly to $P_{\Omega_{1} \cap \Omega_{2}} x_{0}$.

Remark 4.4. Our results in this paper also hold for infinite family of uniformly continuous quasi-nonexpansive mappings in a real Hilbert space. 


\section{References}

[1] R. E. Bruck Jr., "On the weak convergence of an ergodic iteration for the solution of variational inequalities for monotone operators in Hilbert space," Journal of Mathematical Analysis and Applications, vol. 61, no. 1, pp. 159-164, 1977.

[2] F. E. Browder and W. V. Petryshyn, "Construction of fixed points of nonlinear mappings in Hilbert space," Journal of Mathematical Analysis and Applications, vol. 20, pp. 197-228, 1967.

[3] H. Iiduka and W. Takahashi, "Strong convergence theorems for nonexpansive mappings and inversestrongly monotone mappings," Nonlinear Analysis: Theory, Methods E Applications, vol. 61, no. 3, pp. 341-350, 2005.

[4] M. Liu, S. Chang, and P. Zuo, "On a hybrid method for generalized mixed equilibrium problem and fixed point problem of a family of quasi- $\phi$-asymptotically nonexpansive mappings in Banach spaces," Fixed Point Theory and Applications, vol. 2010, Article ID 157278, 18 pages, 2010.

[5] N. Petrot, K. Wattanawitoon, and P. Kumam, "A hybrid projection method for generalized mixed equilibrium problems and fixed point problems in Banach spaces," Nonlinear Analysis: Hybrid Systems, vol. 4, no. 4, pp. 631-643, 2010.

[6] S. Zhang, "Generalized mixed equilibrium problem in Banach spaces," Applied Mathematics and Mechanics. English Edition, vol. 30, no. 9, pp. 1105-1112, 2009.

[7] P. L. Combettes and S. A. Hirstoaga, "Equilibrium programming in Hilbert spaces," Journal of Nonlinear and Convex Analysis, vol. 6, no. 1, pp. 117-136, 2005.

[8] Y. Liu, "A general iterative method for equilibrium problems and strict pseudo-contractions in Hilbert spaces," Nonlinear Analysis: Theory, Methods E Applications, vol. 71, no. 10, pp. 4852-4861, 2009.

[9] A. Moudafi, "Weak convergence theorems for nonexpansive mappings and equilibrium problems," Journal of Nonlinear and Convex Analysis, vol. 9, no. 1, pp. 37-43, 2008.

[10] S. Plubtieng and R. Punpaeng, "A new iterative method for equilibrium problems and fixed point problems of nonexpansive mappings and monotone mappings," Applied Mathematics and Computation, vol. 197, no. 2, pp. 548-558, 2008.

[11] X. Qin, M. Shang, and Y. Su, "Strong convergence of a general iterative algorithm for equilibrium problems and variational inequality problems," Mathematical and Computer Modelling, vol. 48, no. 7-8, pp. 1033-1046, 2008.

[12] Y. Su, M. Shang, and X. Qin, "An iterative method of solution for equilibrium and optimization problems," Nonlinear Analysis: Theory, Methods \& Applications, vol. 69, no. 8, pp. 2709-2719, 2008.

[13] S. Takahashi and W. Takahashi, "Viscosity approximation methods for equilibrium problems and fixed point problems in Hilbert spaces," Journal of Mathematical Analysis and Applications, vol. 331, no. 1, pp. 506-515, 2007.

[14] R. Wangkeeree, "An extragradient approximation method for equilibrium problems and fixed point problems of a countable family of nonexpansive mappings," Fixed Point Theory and Applications, vol. 2008, Article ID 134148, 17 pages, 2008.

[15] Y. J. Cho, X. Qin, and J. I. Kang, "Convergence theorems based on hybrid methods for generalized equilibrium problems and fixed point problems," Nonlinear Analysis: Theory, Methods E Applications, vol. 71, no. 9, pp. 4203-4214, 2009.

[16] X. Qin, Y. J. Cho, and S. M. Kang, "Viscosity approximation methods for generalized equilibrium problems and fixed point problems with applications," Nonlinear Analysis: Theory, Methods $\mathcal{E}$ Applications, vol. 72, no. 1, pp. 99-112, 2010.

[17] Y. Shehu, "Fixed point solutions of generalized equilibrium problems for nonexpansive mappings," Journal of Computational and Applied Mathematics, vol. 234, no. 3, pp. 892-898, 2010.

[18] S. Takahashi and W. Takahashi, "Strong convergence theorem for a generalized equilibrium problem and a nonexpansive mapping in a Hilbert space," Nonlinear Analysis: Theory, Methods E Applications, vol. 69, no. 3, pp. 1025-1033, 2008.

[19] J.-W. Peng and J.-C. Yao, "Strong convergence theorems of iterative scheme based on the extragradient method for mixed equilibrium problems and fixed point problems," Mathematical and Computer Modelling, vol. 49, no. 9-10, pp. 1816-1828, 2009.

[20] S. Plubtieng and K. Sombut, "Weak convergence theorems for a system of mixed equilibrium problems and nonspreading mappings in a Hilbert space," journal of Inequalities and Applications, vol. 2010, Article ID 246237, 12 pages, 2010.

[21] Y. Yao, Y.-C. Liou, and J.-C. Yao, "A new hybrid iterative algorithm for fixed-point problems, variational inequality problems, and mixed equilibrium problems," Fixed Point Theory and Applications, vol. 2008, Article ID 417089, 15 pages, 2008. 
[22] E. Blum and W. Oettli, "From optimization and variational inequalities to equilibrium problems," The Mathematics Student, vol. 63, no. 1-4, pp. 123-145, 1994.

[23] L.-C. Ceng, A. R. Khan, Q. H. Ansari, and J.-C. Yao, "Viscosity approximation methods for strongly positive and monotone operators," Fixed Point Theory, vol. 10, no. 1, pp. 35-72, 2009.

[24] A. Moudafi and M. Théra, "Proximal and dynamical approaches to equilibrium problems," in IllPosed Variational Problems and Regularization Techniques, vol. 477 of Lecture Notes in Economics and Mathematical Systems, pp. 187-201, Springer, Berlin, Germany, 1999.

[25] N. Nadezhkina and W. Takahashi, "Weak convergence theorem by an extragradient method for nonexpansive mappings and monotone mappings," Journal of Optimization Theory and Applications, vol. 128, no. 1, pp. 191-201, 2006.

[26] M. A. Noor, "General variational inequalities and nonexpansive mappings," Journal of Mathematical Analysis and Applications, vol. 331, no. 2, pp. 810-822, 2007.

[27] P. Kumam and P. Katchang, "A viscosity of extragradient approximation method for finding equilibrium problems, variational inequalities and fixed point problems for nonexpansive mappings," Nonlinear Analysis: Hybrid Systems, vol. 3, no. 4, pp. 475-486, 2009.

[28] S. Plubtieng and P. Kumam, "Weak convergence theorem for monotone mappings and a countable family of nonexpansive mappings," Journal of Computational and Applied Mathematics, vol. 224, no. 2, pp. 614-621, 2009.

[29] X. Qin, Y. J. Cho, and S. M. Kang, "Convergence theorems of common elements for equilibrium problems and fixed point problems in Banach spaces," Journal of Computational and Applied Mathematics, vol. 225, no. 1, pp. 20-30, 2009.

[30] A. Tada and W. Takahashi, "Weak and strong convergence theorems for a nonexpansive mapping and an equilibrium problem," Journal of Optimization Theory and Applications, vol. 133, no. 3, pp. 359-370, 2007.

[31] W. Takahashi and M. Toyoda, "Weak convergence theorems for nonexpansive mappings and monotone mappings," Journal of Optimization Theory and Applications, vol. 118, no. 2, pp. 417-428, 2003.

[32] Y. Yao and J.-C. Yao, "On modified iterative method for nonexpansive mappings and monotone mappings," Applied Mathematics and Computation, vol. 186, no. 2, pp. 1551-1558, 2007.

[33] J. Zhao and S. He, "A new iterative method for equilibrium problems and fixed point problems of infinitely nonexpansive mappings and monotone mappings," Applied Mathematics and Computation, vol. 215, no. 2, pp. 670-680, 2009.

[34] B. Halpern, "Fixed points of nonexpanding maps," Bulletin of the American Mathematical Society, vol. 73, pp. 957-961, 1967.

[35] P.-L. Lions, "Approximation de points fixes de contractions," Comptes Rendus de l'Académie des Sciences Paris A-B, vol. 284, no. 21, pp. A1357-A1359, 1977.

[36] R. Wittmann, "Approximation of fixed points of nonexpansive mappings," Archiv der Mathematik, vol. 58, no. 5, pp. 486-491, 1992.

[37] S. Reich, "Strong convergence theorems for resolvents of accretive operators in Banach spaces," Journal of Mathematical Analysis and Applications, vol. 75, no. 1, pp. 287-292, 1980.

[38] N. Shioji and W. Takahashi, "Strong convergence of approximated sequences for nonexpansive mappings in Banach spaces," Proceedings of the American Mathematical Society, vol. 125, no. 12, pp. 3641-3645, 1997.

[39] C. Martinez-Yanes and H.-K. Xu, "Strong convergence of the CQ method for fixed point iteration processes," Nonlinear Analysis: Theory, Methods \& Applications, vol. 64, no. 11, pp. 2400-2411, 2006.

[40] S. Plubtieng and K. Ungchittrakool, "Strong convergence theorems for a common fixed point of two relatively nonexpansive mappings in a Banach space," Journal of Approximation Theory, vol. 149, no. 2, pp. 103-115, 2007.

[41] X. Qin and Y. Su, "Strong convergence theorems for relatively nonexpansive mappings in a Banach space," Nonlinear Analysis: Theory, Methods E Applications, vol. 67, no. 6, pp. 1958-1965, 2007.

[42] X. L. Qin, Y. J. Cho, S. M. Kang, and H. Y. Zhou, "Convergence of a hybrid projection algorithm in Banach spaces," Acta Applicandae Mathematicae, vol. 108, no. 2, pp. 299-313, 2009.

[43] R. Wangkeeree and R. Wangkeeree, "The shrinking projection method for solving variational inequality problems and fixed point problems in Banach spaces," Abstract and Applied Analysis, vol. 2009, Article ID 624798, 26 pages, 2009.

[44] L.-C. Ceng and J.-C. Yao, "A hybrid iterative scheme for mixed equilibrium problems and fixed point problems," Journal of Computational and Applied Mathematics, vol. 214, no. 1, pp. 186-201, 2008. 
[45] M. Liu, S. S. Chang, and P. Zuo, "An algorithm for finding a common solution for a system of mixed equilibrium problem, quasivariational inclusion problem, and fixed point problem of nonexpansive semigroup," Journal of Inequalities and Applications, vol. 2010, Article ID 895907, 23 pages, 2010.

[46] Y. Yao, M. A. Noor, S. Zainab, and Y.-C. Liou, "Mixed equilibrium problems and optimization problems," Journal of Mathematical Analysis and Applications, vol. 354, no. 1, pp. 319-329, 2009.

[47] R. Wangkeeree and R. Wangkeeree, "A general iterative method for variational inequality problems, mixed equilibrium problems, and fixed point problems of strictly pseudocontractive mappings in Hilbert spaces," Fixed Point Theory and Applications, vol. 2009, Article ID 519065, 32 pages, 2009.

[48] W. Takahashi, Y. Takeuchi, and R. Kubota, "Strong convergence theorems by hybrid methods for families of nonexpansive mappings in Hilbert spaces," Journal of Mathematical Analysis and Applications, vol. 341, no. 1, pp. 276-286, 2008.

[49] P. Kumam, "A new hybrid iterative method for solution of equilibrium problems and fixed point problems for an inverse strongly monotone operator and a nonexpansive mapping," Journal of Applied Mathematics and Computing, vol. 29, no. 1-2, pp. 263-280, 2009.

[50] W. Chantarangsi, C. Jaiboon, and P. Kumam, "A viscosity hybrid steepest descent method for generalized mixed equilibrium problems and variational inequalities for relaxed cocoercive mapping in Hilbert spaces," Abstract and Applied Analysis, vol. 2010, Article ID 390972, 39 pages, 2010.

[51] J.-B. Baillon and G. Haddad, "Quelques propriétés des opérateurs angle-bornés et $n$-cycliquement monotones," Israel Journal of Mathematics, vol. 26, no. 2, pp. 137-150, 1977.

[52] W. Takahashi, Nonlinear Functional Analysis, Fixed Point Theory and Its Application, Yokohama Publishers, Yokohama, Japan, 2000. 


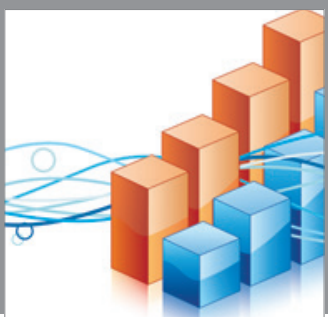

Advances in

Operations Research

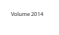

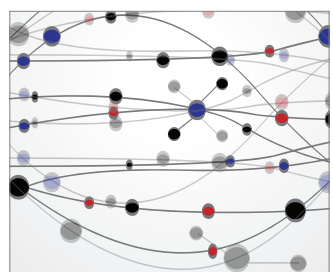

\section{The Scientific} World Journal
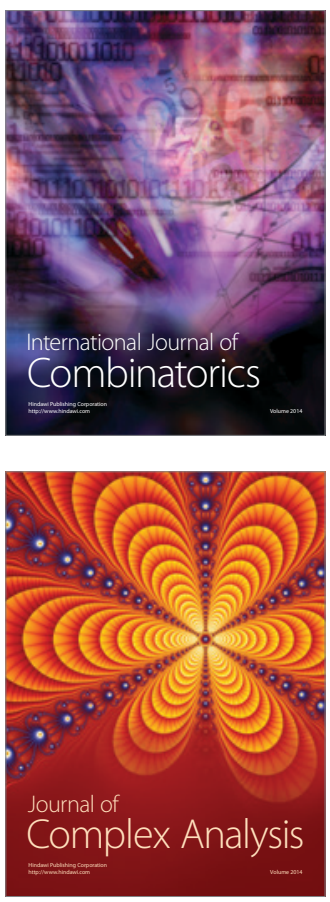

International Journal of

Mathematics and

Mathematical

Sciences
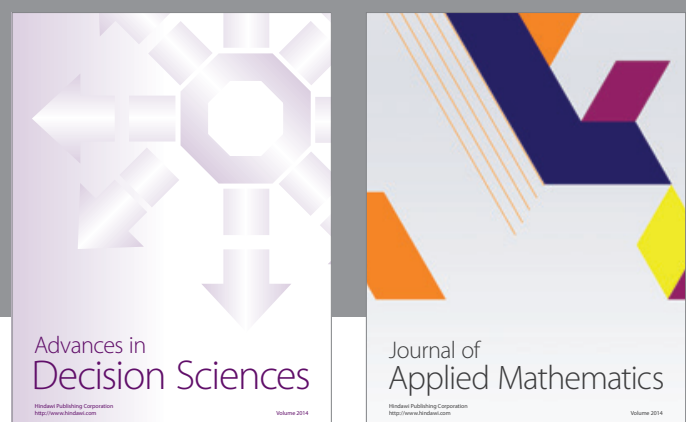

Journal of

Applied Mathematics
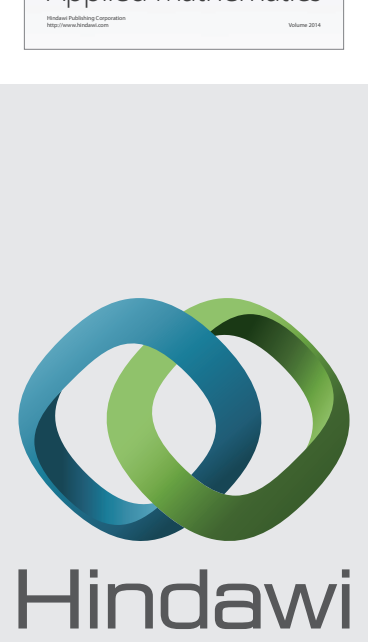

Submit your manuscripts at http://www.hindawi.com
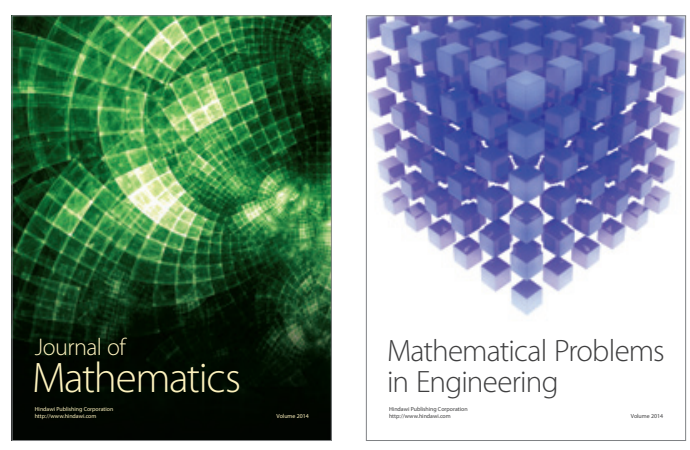

Mathematical Problems in Engineering
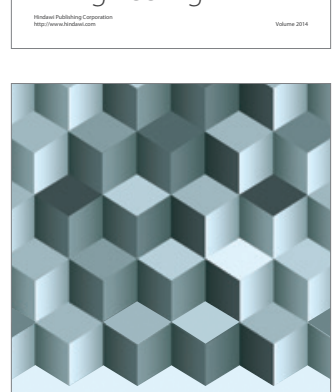

Journal of

Function Spaces
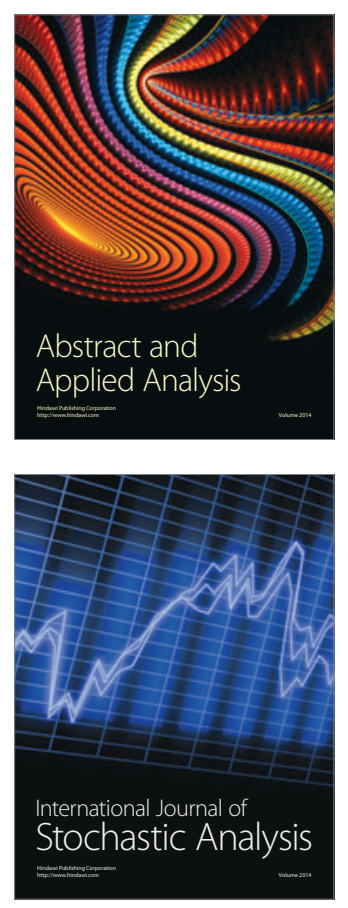

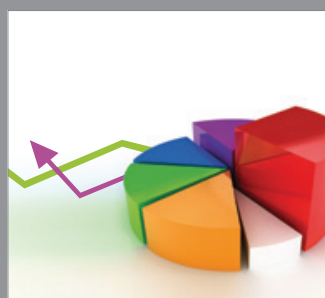

ournal of

Probability and Statistics

Promensencen
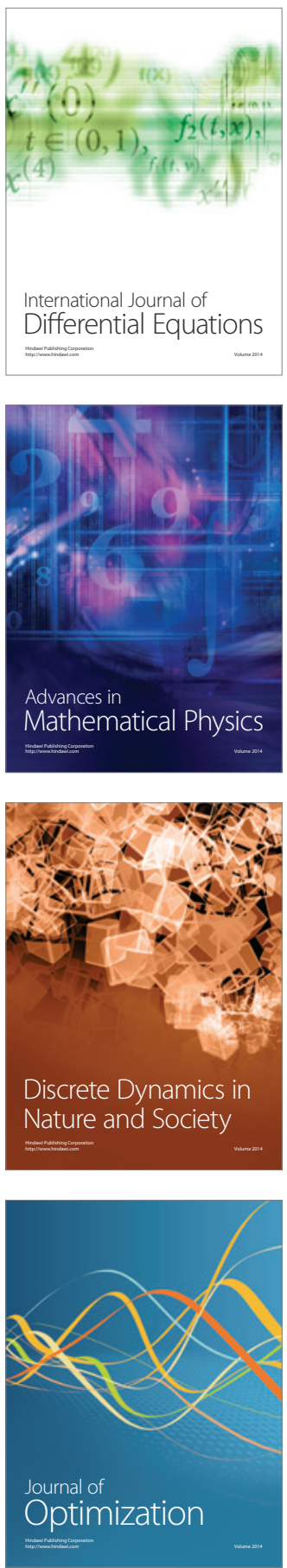